\title{
The association between Alu hypomethylation and severity of type 2 diabetes mellitus
}

\author{
Jirapan Thongsroy ${ }^{1 *}$ D , Maturada Patchsung ${ }^{2}$ and Apiwat Mutirangura 3,4
}

\begin{abstract}
Background: Cellular senescence due to genomic instability is believed to be one of the mechanisms causing health problems in diabetes mellitus (DM). Low methylation levels of Alu elements or Alu hypomethylation, an epigenomic event causing genomic instability, were commonly found in aging people and patients with aging phenotypes, such as osteoporosis.

Results: We investigate Alu methylation levels of white blood cells of type $2 \mathrm{DM}$, pre-DM, and control. The DM group possess the lowest Alu methylation $(P<0.001, P<0.0001$ adjusted age). In the DM group, Alu hypomethylation is directly correlated with high fasting blood sugar, $\mathrm{HbA1C}$, and blood pressure.

Conclusion: Genome-wide hypomethylation may be one of the underlining mechanisms causing genomic instability in type 2 DM. Moreover, Alu methylation levels may be a useful biomarker for monitoring cellular senescence in type 2 DM patients.
\end{abstract}

Keywords: DNA methylation, Diabetes mellitus, Genomic instability, Alu, Senescence

\section{Background}

Diabetes mellitus or DM is a complex multifactorial disorder in which the person has high blood glucose (hyperglycemia) [1,2]. Type $2 \mathrm{DM}$ is the most common type of diabetes. At present, an estimated 387 million people have DM, and that number is expected to reach 592 million by 2035 [3]. DM patients possess increased risk of various geriatric conditions; therefore, type $2 \mathrm{DM}$ is one of the most serious health problems in the world [4-7]. Cellular senescence, particularly vascular senescence, is believed to be a main contributing factor to DM-related complications, such as retinopathy, kidney failure, cerebrovascular disease, and delay wound healing $[8,9]$. Nevertheless, the cellular senescence mechanism in DM remains to be explored.

A low Alu methylation level, Alu hypomethylation, has been reported to represent a cellular senescence biomarker $[10,11]$. There are more than 1 million copies of Alu elements, a class of short intersperse elements [12].

\footnotetext{
* Correspondence: ju_jirapan@hotmail.com

${ }^{1}$ School of Medicine, Walailak University, Nakhon Si Thammarat, Thailand Full list of author information is available at the end of the article
}

Alu elements involve up to $11 \%$ of the human genome [13]. Alu hypomethylation was first found in white blood cells (WBCs) of aging people during ages 34-68 years $[10,14]$. In contrast to aging, higher Alu methylation levels were found in individuals who had catch-up growth during newborn periods [15]. Alu hypomethylation may play a role in the senescence process. When age is adjusted, Alu hypomethylation is independently associated with lower bone mass density including osteopenia and osteoporosis and a high body mass index [11]. Therefore, it is interesting to explore whether cellular senescence of DM also correlates with Alu hypomethylation. Many studies concluded that genome-wide hypomethylation can promote genomic instability $[16,17]$. This study will provide information about whether or not global hypomethylation promoting genomic instability is involved in DM pathogenesis.

To investigate Alu methylation changes during type 2 DM development, we measured the level of Alu methylation in normal, pre-type $2 \mathrm{DM}$, and type $2 \mathrm{DM}$ patients by ALU-Combined Bisulfite Restriction Analysis. COBRA-interspersed repetitive sequence PCR is a highly accurate quantitative methylation measurement. Where 
Table 1 Sample size, age, and body mass index (BMI) in each group by FBS and HbA1C indicator

\begin{tabular}{|c|c|c|c|c|}
\hline & Group & & & $P$ value \\
\hline & Normal & Pre-DM & DM & \\
\hline FBS indicator & & & & \\
\hline$n$ & 80 & 80 & 80 & \\
\hline Sex & & & & \\
\hline Male & $15(18.75 \%)$ & $12(15.00 \%)$ & $24(30.00 \%)$ & \\
\hline Female & $65(81.25 \%)$ & $68(85.00 \%)$ & $56(70.00 \%)$ & \\
\hline Age (years) (mean $\pm S D$ ) & $50.04 \pm 9.65$ & $51.21 \pm 9.27$ & $59.99 \pm 11.88$ & $<0.001$ \\
\hline $\mathrm{BMI}\left(\mathrm{kg} / \mathrm{m}^{2}\right)($ mean $\pm \mathrm{SD})$ & $25.04 \pm 3.81$ & $25.64 \pm 3.97$ & $27.17 \pm 9.89$ & 0.0984 \\
\hline $\mathrm{HbA} 1 \mathrm{C}$ indicator & & & & \\
\hline$n$ & 42 & 113 & 85 & \\
\hline Sex & & & & \\
\hline Male & $5(11.90 \%)$ & $28(24.78 \%)$ & $11(12.94 \%)$ & \\
\hline Female & $37(88.10 \%)$ & 85 (75.22\%) & $74(87.06 \%)$ & \\
\hline Age (years) (mean \pm SD) & $47.67 \pm 9.03$ & $53.64 \pm 10.67$ & $56.89 \pm 11.73$ & $<0.001$ \\
\hline $\mathrm{BMI}\left(\mathrm{kg} / \mathrm{m}^{2}\right)($ mean $\pm \mathrm{SD})$ & $23.82 \pm 3.66$ & $26.45 \pm 7.94$ & $26.31 \pm 5.07$ & 0.0627 \\
\hline
\end{tabular}

pyrosequencing cannot demonstrate DNA methylation pattern, COBRA, detecting more than one CpG site, can (Additional file 1: Figure S1). In many cases, the DNA methylation pattern was more sensitive in revealing DNA methylation changes than DNA methylation levels alone [18-20].

\section{Methods}

\section{Participants}

For the study, we used 240 cases that were being monitored by fasting blood sugar (FBS) levels and were classified into three groups: normal (80 samples), pre-DM (80 samples), and DM (80 samples). Pre-DM and DM were admitted to the Tambon Health Promoting Hospital, Thailand, between 2015 and 2016. Sample ages were between 15 and 80 years. All subjects voluntarily participated in the study. The study was reviewed and approved by the Ethics Clearance Committee on Human
Rights Related to Research Involving Human Subjects, Walailak University, Nakhon Si Thammarat, Thailand. Written informed consent was obtained from each participant.

\section{DNA extraction and bisulfite DNA modification}

DNA was extracted from buffy coat using proteinase $\mathrm{K}$ digestion and phenol-chloroform extraction protocols. Bisulfite treatment was performed as per standard protocols, with some modifications. Briefly, denatured genomic DNA was incubated in $0.22 \mathrm{M} \mathrm{NaOH}$ at $37{ }^{\circ} \mathrm{C}$ for $10 \mathrm{~min}$, followed by addition of $30 \mu \mathrm{l}$ of $10 \mathrm{mM}$ hydroquinone and $520 \mu \mathrm{l}$ of $3 \mathrm{M}$ sodium-bisulfite for $16-20 \mathrm{~h}$ at $50{ }^{\circ} \mathrm{C}$. Subsequently, the DNA was purified and incubated in $0.33 \mathrm{M} \mathrm{NaOH}$ at $25{ }^{\circ} \mathrm{C}$ for 3 min, ethanol precipitated, washed with $70 \%$ ethanol, and resuspended in $20 \mu \mathrm{l}$ of $\mathrm{H}_{2} \mathrm{O}$.
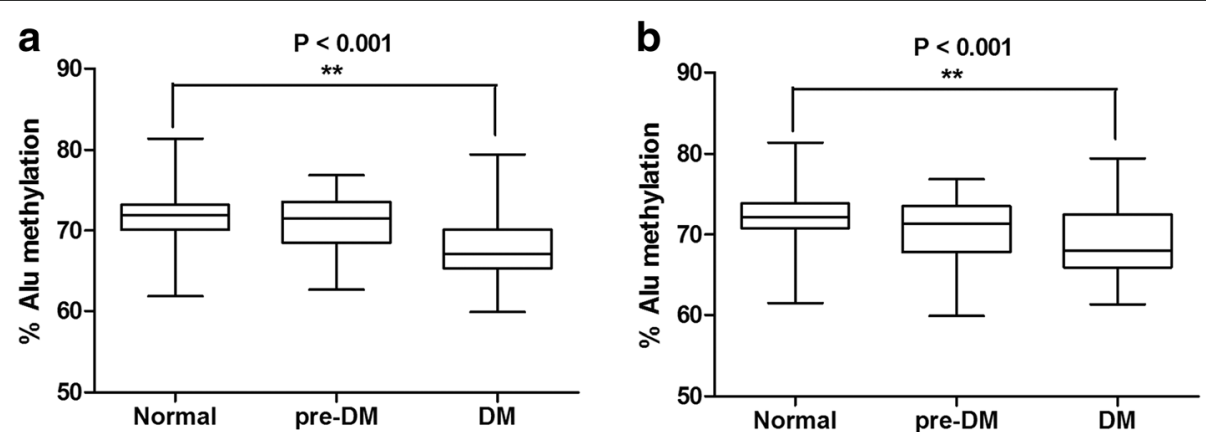

Fig. 1 The percentage of each Alu methylation level in normal, pre-DM, and DM samples grouped by fasting blood sugar level (a) and hemoglobin A1C ( $\mathrm{HbA1C}$ ) (b). Boxes represent interquartile ranges (25th to 75th percentile) and median lines represent the 50th percentile. The whiskers represent minimum and maximum values. ${ }^{*} P<0.05,{ }^{*} P<0.001$ (Mann-Whitney test) 


\section{ALU-Combined Bisulfite Restriction Analysis (COBRA)}

To observe methylation levels of Alu in samples, the sodium-bisulfite-treated DNA in each sample was amplified by PCR containing 1x PCR buffer (Qiagen, Germany), $0.2 \mathrm{mM}$ of deoxynucleotide triphosphate (Promega, USA), $1 \mathrm{mM}$ of magnesium chloride (Qiagen, Germany), $25 \mathrm{U}$ of HotStarTaq DNA Polymerase (Qiagen, Germany), and $0.3 \mu \mathrm{M}$ primer pairs: ALU-BRev (5'-CTAACTTTTTA TATTTTTAATAAAAACRAAATTTCAC CA-3') where $\mathrm{R}=\mathrm{A}$ and $\mathrm{G}$ and $\mathrm{Y}=\mathrm{C}$ and $\mathrm{T}$. For Alu amplification, the program was set as follows: $95^{\circ} \mathrm{C}$ for $15 \mathrm{~min}, 40$ cycles of $95{ }^{\circ} \mathrm{C}$ for $45 \mathrm{~s}, 57^{\circ} \mathrm{C}$ for $45 \mathrm{~s}$, and $72{ }^{\circ} \mathrm{C}$ for $45 \mathrm{~s}$, followed by a final extension of $72{ }^{\circ} \mathrm{C}$ for $7 \mathrm{~min}$ [20]. Alu PCR products were subjected to COBRA using $2 \mathrm{U}$ of TaqI
(Thermo scientific, USA), $2 \mathrm{U}$ of TasI (Thermo scientific, USA), 5x NEB3 buffer (New England Biolabs, USA), and $1 \mu \mathrm{g} / \mathrm{ul}$ bovine serum albumin (BSA) (New England Biolabs, USA) and incubated at $65{ }^{\circ} \mathrm{C}$ overnight. The cut PCR products were analyzed by $8 \%$ acrylamide gel and SYBR stain (Lonza, USA). The band intensity of Alu methylation was observed and measured by typhoon fla 7000 and ImageQuanNT Software (Amersham biosciences, UK) (Additional file 1: Figure S1) [11].

\section{Methylation analysis}

ALU methylation analysis consisted of calculating the band intensity of the five Alu product sizes; 133, 90, 75, 58, and $43 \mathrm{bp}$ according to the formula: $A=133 / 133$,
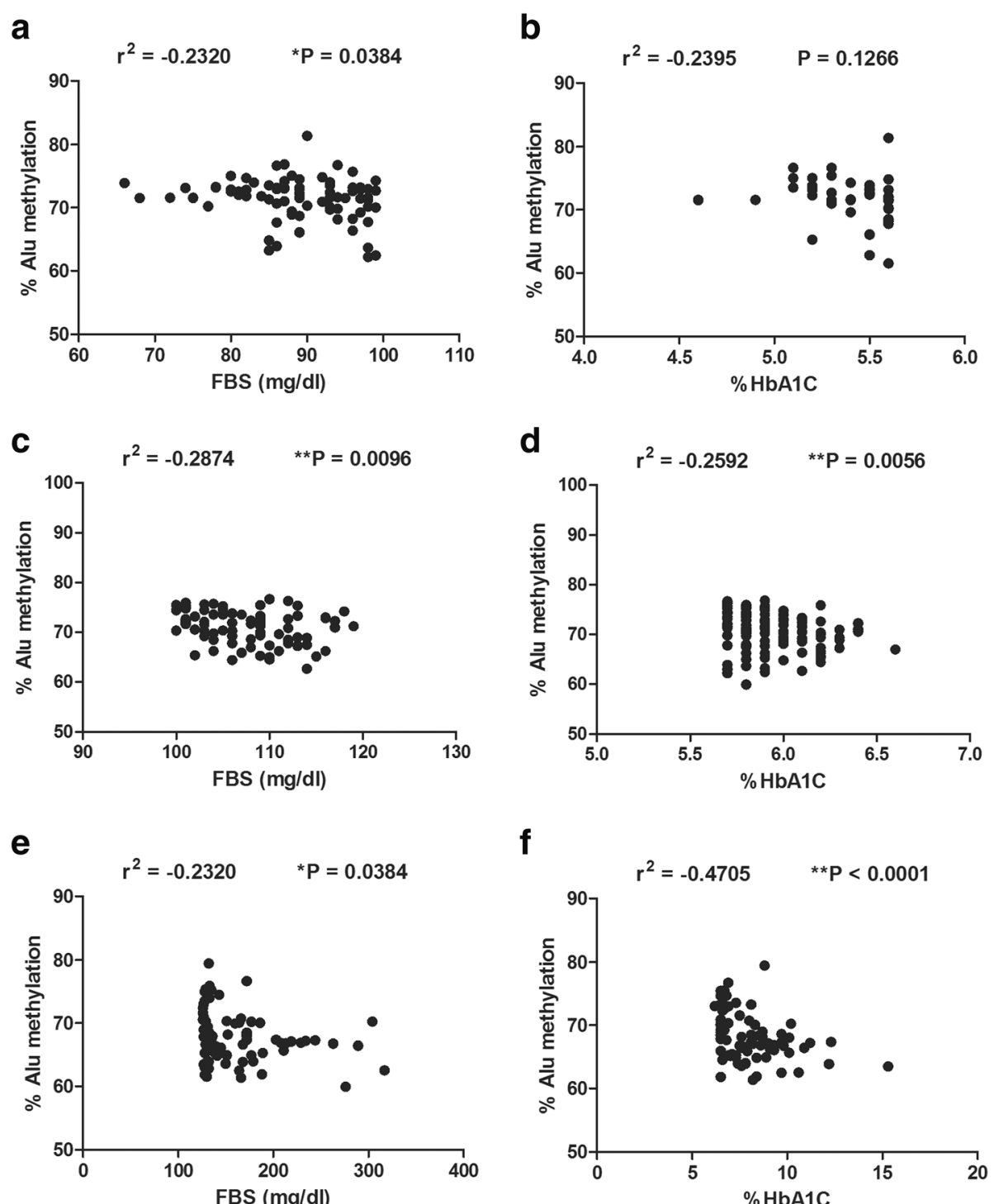

Fig. 2 Association between Alu methylation with FBS and $\mathrm{HbA1C}$ levels. Correlation between \% Alu methylation and FBS in normal (a) pre-DM $(\mathbf{b})$, and DM (c). Correlation between Alu methylation with HbA1C in normal (d), pre-DM (e), and DM (f). Pearson's correlation coefficients $(r)$ with $P$ values are indicated $\left({ }^{*} P<0.05,{ }^{*} P<0.001\right)$ 

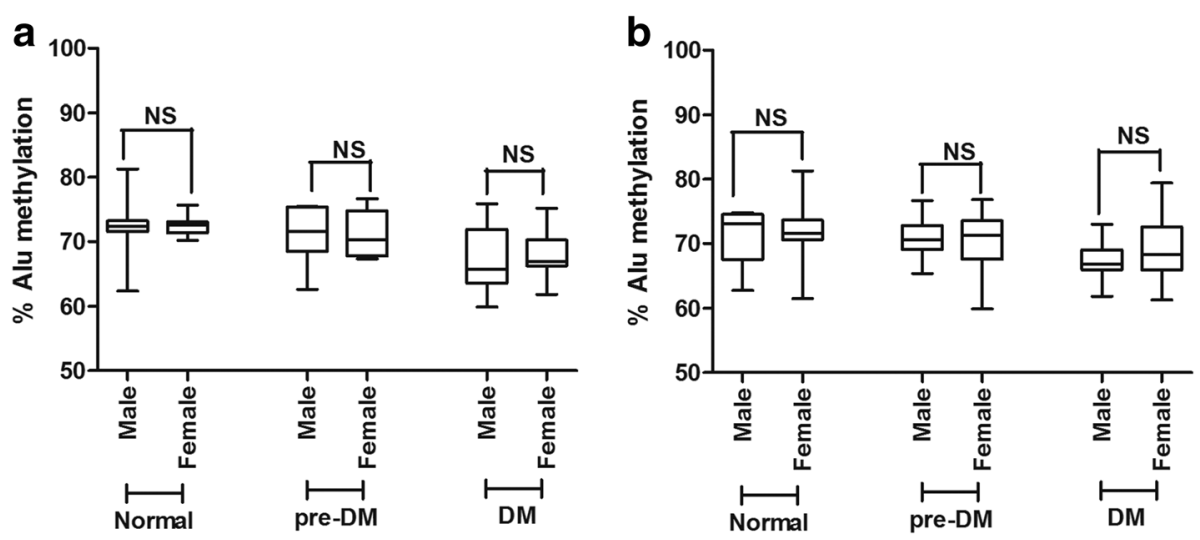

Fig. 3 Comparisons of Alu methylation levels between male and female in normal, pre-DM, and DM when grouped using FBS indicator (a) and $\mathrm{HbA1C}$ indicator (b). The boxes represent interquartile ranges (25th to 75th percentile) and median lines represent the 50th percentile. The whiskers represent minimum and maximum values. ${ }^{*} P<0.05$, ${ }^{*} P<0.001$ (Mann-Whitney test)

$B=58 / 58, C=75 / 75, D=90 / 90, E=43 / 43$, and $F=[(E+B)-(C+D)] / 2$. The Alu methylation level was calculated from $[(E+B) \times 100] /(2 \mathrm{~A}+E+B+C+D)$.

\section{Statistical analyses}

Data was analyzed with SPSS statistical software. The average and distributions of characteristic data of type 2 $\mathrm{DM}$ are presented as the mean \pm SD and median. Pearson's correlation between the Alu methylation pattern and characteristic data of the population were used to determine $95 \%$ confidence intervals of association. $T$ score was used to divide the subjects into three groups: normal, pre-DM, and DM. T-test was used to determine the differences using a $P$ value threshold of 0.05 between the groups in the matched cases based on fasting blood sugar (FBS). Logistic regression models were used to evaluate the association between FBS by $T$-score as a dependent variable specified as a binary outcome, normal and $\mathrm{DM}$ and \%mC, \% $\mathrm{mCmC}$ loci, \% $\mathrm{uCmC}$ loci,
$\% \mathrm{mCuC}$ loci, and \%uCuC loci as independent predictors. All independent variables were used in the same model.

\section{Results}

Alu methylation in patients with type 2 diabetes

Fasting blood sugar (FBS) levels were used to classify the 240 samples into three groups: 80 normal controls, 80 pre-DM, and 80 type 2 DM. These samples were also classified using $\mathrm{HbA} 1 \mathrm{C}$ levels resulting in several patients changing groups (42 normal, 113 pre-DM, and $85 \mathrm{DM}$ ) compared to the values from the FBS indicator (Table 1). Next, we used the ALUCombined Bisulfite Restriction Analysis (COBRA-Alu) to measure Alu methylation in each sample. When comparing Alu methylation levels of the normal with the pre-DM and type $2 \mathrm{DM}$ patients grouped according to FBS, we found that the level of Alu methylation was lowest in type $2 \mathrm{DM}(P<0.001)$ (Fig. 1a).
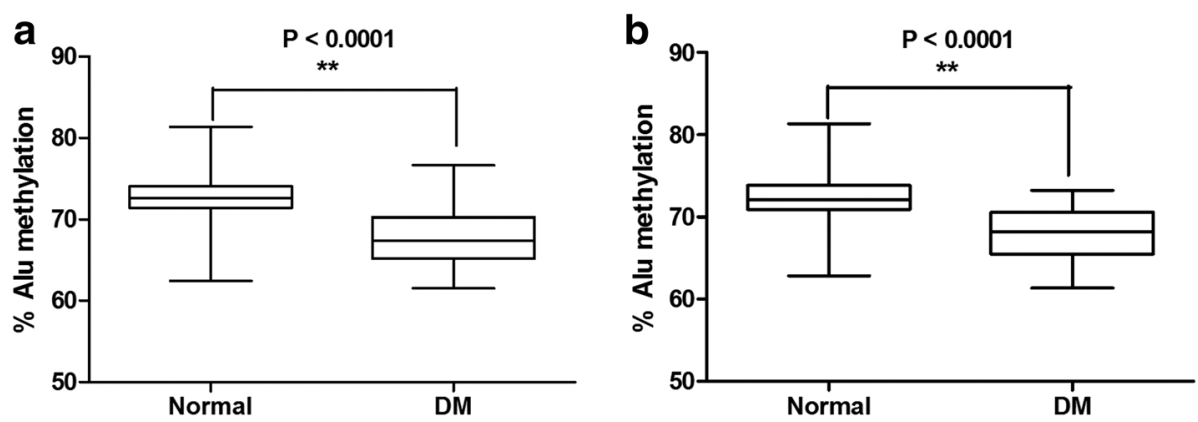

Fig. 4 The percentage of Alu methylation in age-matched pairs between normal samples and DM samples in both the FBS (a) and HbA1C groups (b). The values from 30 independent age-matched pairs are shown as box plots, with the boxes representing interquartile ranges (25th to 75th percentile) and median lines representing the 50th percentile. The whiskers represent minimum and maximum values. ${ }^{*} P<0.05$, ${ }^{* *} P<0.001$ (Mann-Whitney test) 
Similarly, when grouped by the HbA1C indicator, we found significantly decreased Alu methylation levels in type $2 \mathrm{DM}$ when compared with normal $(P<0.001)$ (Fig. 1b).

Correlations between Alu methylation and FBS or HbA1C in normal, pre-DM, and type 2 DM are reported (Fig. 2 a-f). All groups showed higher biochemical markers of DM, FBS or $\mathrm{HbA1C}$, and lower Alu methylation levels (Fig. 2 a-f). Moreover, the most striking comparison was the correlation between Alu methylation and $\mathrm{HbA1C}$ in $\mathrm{DM}\left(r^{2}=-0.4705\right.$, $P<0.0001$ ) (Fig. 2f).
Age- and sex-adjusted correlation

To determine if sex influenced Alu methylation levels, significant differences in Alu methylation levels were detected between males and females. Here, we divided males and females into normal, pre-DM, and type $2 \mathrm{DM}$ groups. Alu methylation levels were not significantly different between males and females in either FBS (Fig. 3a) or HbA1C groups (Fig. $3 \mathrm{~b}$ ). This is consistent with previous works that have shown that sex does not affect Alu methylation [10, 15].

The average age in normal and type 2 DM groups was not the same in our data (Table 1). To adjust for age

\section{FBS}
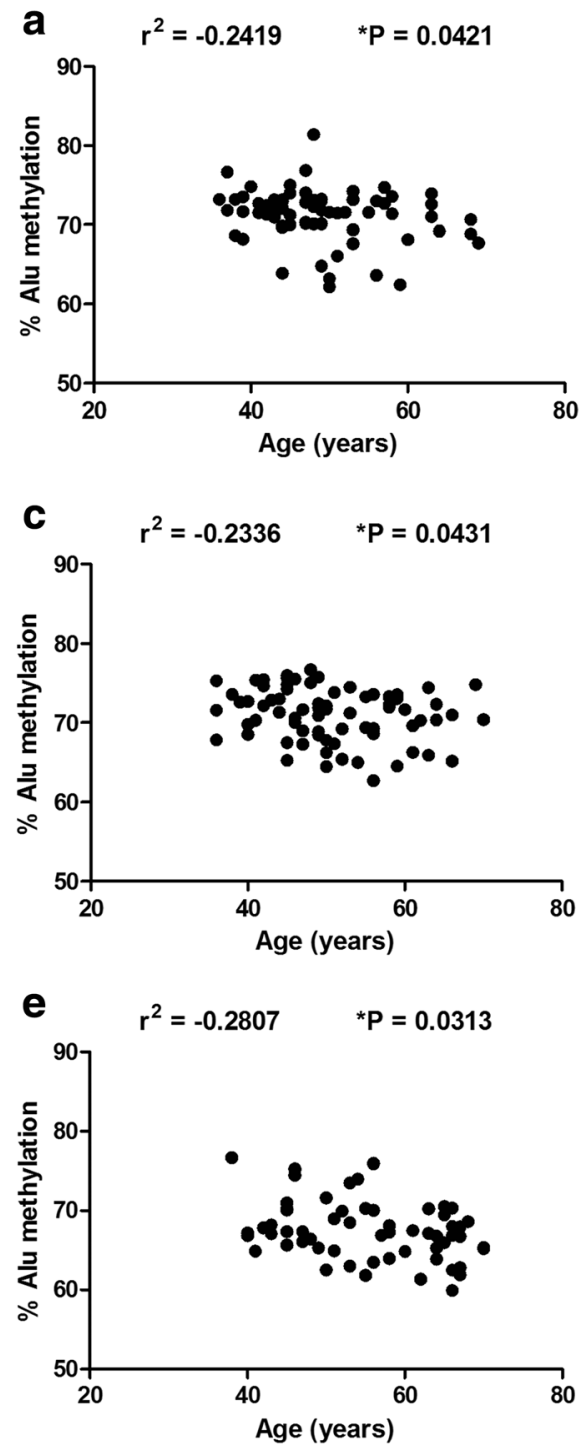

\section{HbA1C}
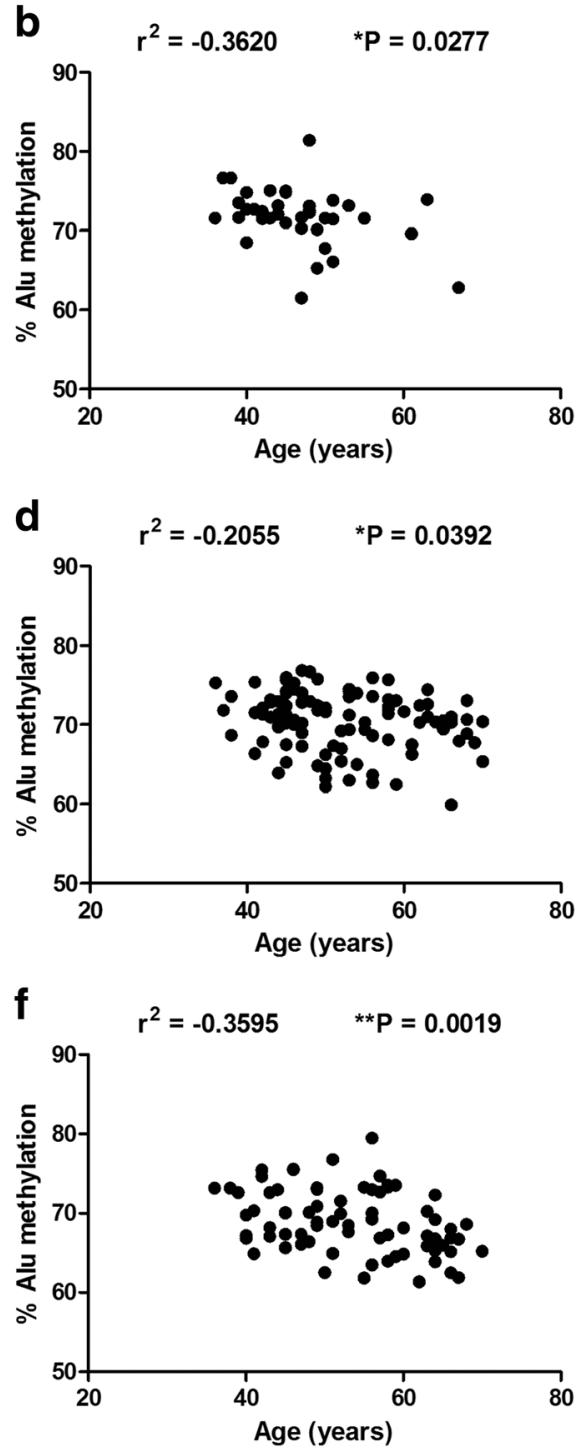

Fig. 5 Correlation between Alu methylation and age in the FBS and $\mathrm{HbA1C}$ groups. Correlation between \% Alu methylation and age in normal (a) pre-DM (b), and DM (c) for the FBS group. Association between Alu methylation with age grouped by HbA1C for normal (d), pre-DM (e), and DM (f). Each plot represents Alu methylation levels of sample. Pearson's correlation coefficients ( $r$ ) with $P$ values are indicated $\left({ }^{*} P<0.05,{ }^{* *} P<0.001\right)$ 
difference, normal samples were matched to same-age DM samples to produce 30 age-matched pairs. The agematched pairs showed significantly decreased Alu methylation in DM compared with normal in both the FBS $(P<0.001)$ and HbA1C groups $(P<0.001)$ (Fig. 4 a, $\mathrm{b}$, respectively). Results were similar to findings before age adjustment (Fig. 1a, b).

A significant negative correlation between Alu methylation and age was observed in all groups (normal, preDM, and DM) when using FBS (Fig. 5a-e) $\left(r^{2}=-0.2419\right.$, $P=0.0421, r^{2}=-0.2336, P=0.0431$, and $r^{2}=-0.2807$, $P=0.0313$, respectively) and $\mathrm{HbA} 1 \mathrm{C}$ (Fig. 5b-f) $\left(r^{2}=-0.3620, P=0.0277, r^{2}=-0.2055, P=0.0392\right.$, and $r^{2}=-0.2320, P=0.0019$, respectively).
Correlation between Alu methylation and hypertension in normal, pre-DM, and type 2 DM

Hypertension in type $2 \mathrm{DM}$ is believed to be one of the severe consequences of vascular senescence [8, 21, 22]. We investigated the relationship between Alu methylation levels and blood pressure (systolic and diastolic) for FBS and HbA1C groups. The results showed a significantly negative association between Alu methylation and systolic pressure only in type $2 \mathrm{DM}$ patients when classified by HbA1C (Fig. 6f). Intriguingly, a similar negative correlation between Alu methylation and diastolic pressure was observed in only type $2 \mathrm{DM}$ patients for the HbA1C group (Fig. 7f). There was no significant correlation between Alu methylation and hypertension in

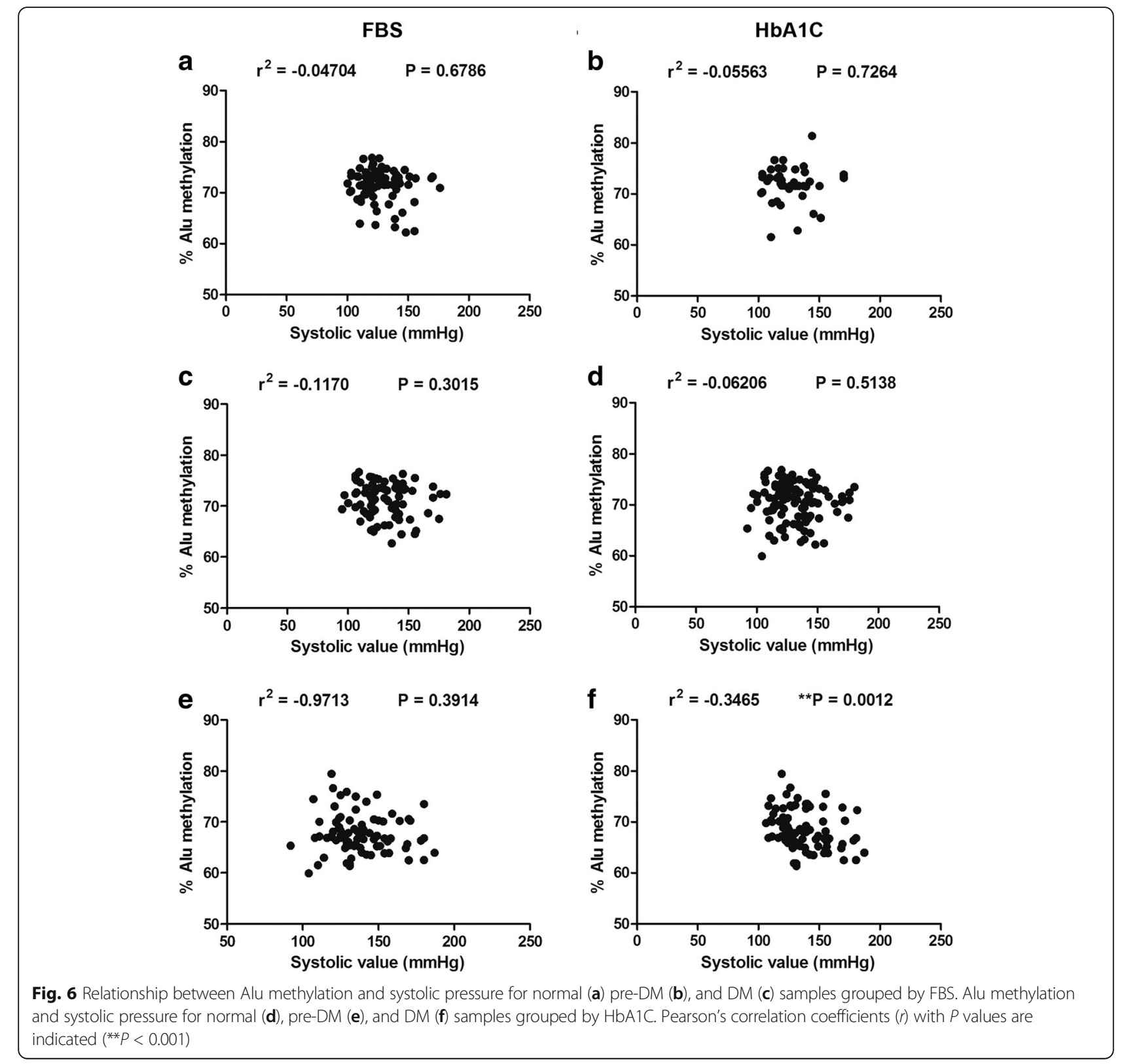


FBS
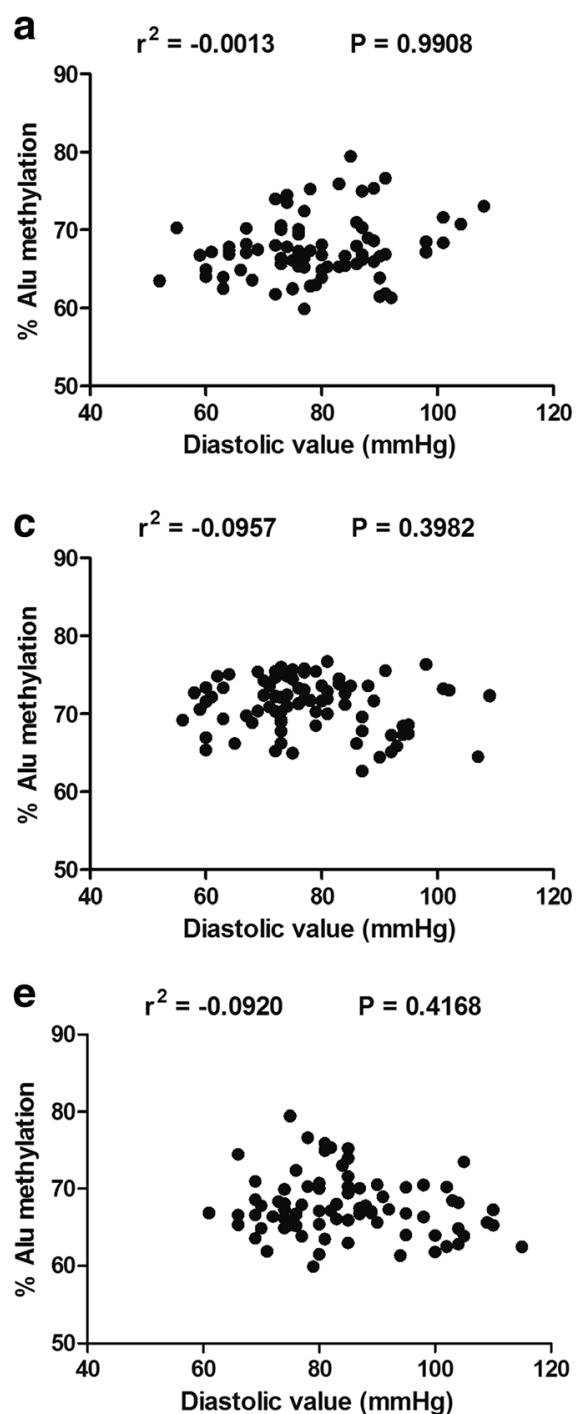

HbA1C
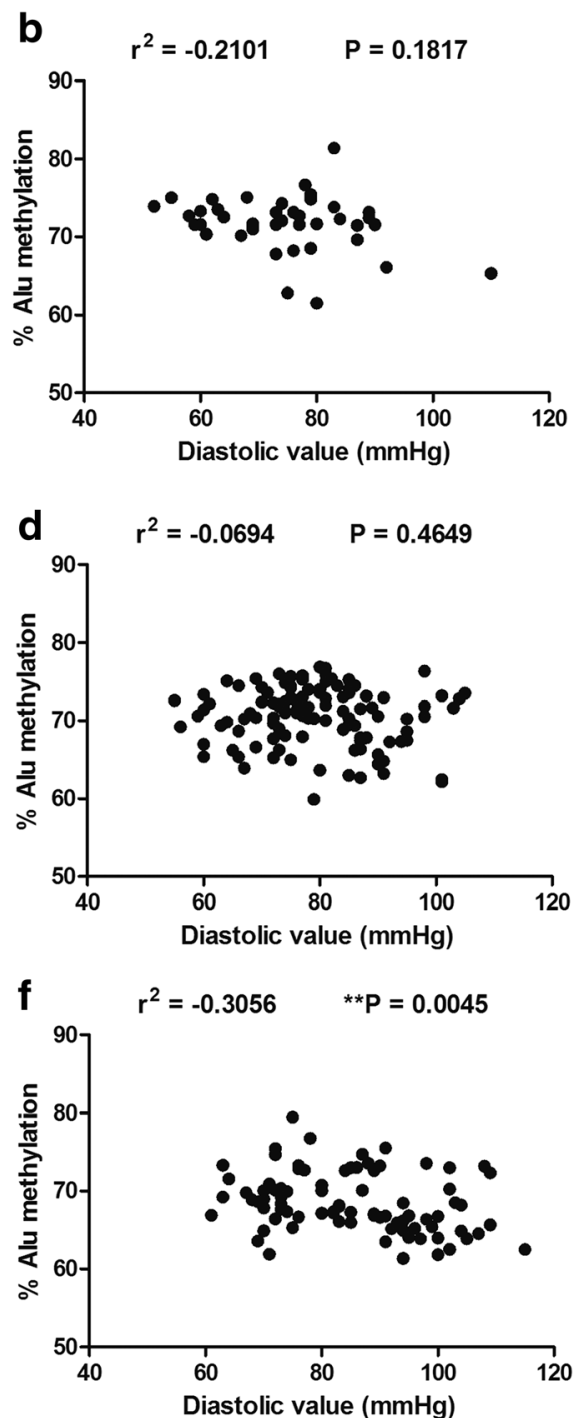

Fig. 7 Association between Alu methylation and diastolic pressure for normal (a), pre-DM (b), and DM (c) samples grouped by FBS. Alu methylation and diastolic pressure for normal (d), pre-DM (e), and DM (f) samples grouped by HbA1C. Pearson's correlation coefficients $(r)$ with $P$ values are indicated $(* * P<0.001)$

normal or pre-DM samples in either the FBS (Figs. 6a, c and $7 \mathrm{a}, \mathrm{c}$ ) or HbA1C groups (Figs. 6b, d and 7b, d).

To prove that the correlation between Alu hypomethylation and hypertension was not influenced by age, we performed age-adjusted experiments (Fig. 8 a, b). Interestingly, the significant correlation was revealed in the DM using $\mathrm{HbA1C}$ as indicator $(P=0.0312)$ (Fig. 8a).

\section{Discussion}

Cellular dysfunction due to aging process was proposed to be one of the pathogenic mechanisms of type $2 \mathrm{DM}[8,9]$. One known mechanism of cellular aging is decrement of genome-wide methylationpromoting genomic instability $[17,23]$. Therefore, in this study, we proved that Alu methylation in type 2 DM patients was lower than that in the general population. Although we found significant differences between DM phenotype and Alu methylation level, there is a significant overlap between Alu methylation levels of DM cases and controls. Moreover, there is no significant difference between pre-DM and normal controls. These limit the use of using Alu methylation levels directly as a biomarker for clinical application. Further study should be performed to evaluate how the change of Alu methylation level of each individual is correlated with progression of the diseases.

Interestingly, we demonstrated a direct correlation between Alu hypomethylation levels with high blood 

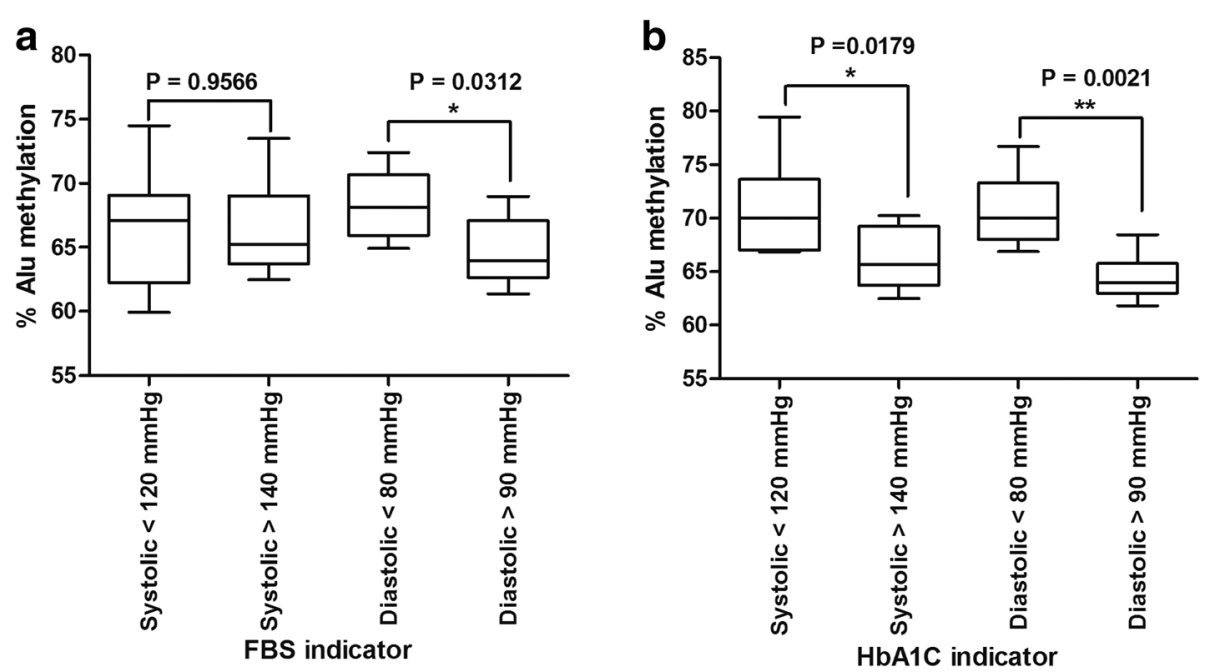

Fig. 8 The percentage of Alu methylation levels in type 2 DM between high systolic/diastolic and normal to adjusted for age when grouped using FBS indicator (a) and $\mathrm{HbA1C}$ indicator (b). The boxes represent interquartile ranges (25th to 75th percentile) and median lines represent the 50th percentile. The whiskers represent minimum and maximum values. ${ }^{*} P<0.05$, ${ }^{* *} P<0.001$ (Mann-Whitney test)

pressure in DM samples when grouped by HbA1C. This association is similar to the correlation between Alu hypomethylation and lower bone mass [11]. Moreover, the fact that the correlation was found only when grouping DM by HbA1C reflected that the finding was a permanent change. Target cellular defects causing high blood pressure in DM are not WBC. The underlining reason of how Alu hypomethylation in WBC is associated with high blood pressure may be that the mechanism causing Alu hypomethylation in DM is systemic and methylation levels of other tissues remained to be explored. Nevertheless, the fact that Alu hypomethylation in WBC is associated with several geriatric degenerative diseases suggests that Alu hypomethylation levels in white blood cells are a promising biomarker for senescence monitoring.

Mechanisms of aberrant methylation and genomic instability in cellular senescence and type $2 \mathrm{DM}$ are unclear. It is possible that oxidative stress products increase glutathione levels that could influence epigenetic processes including DNA and histone methylation by limiting the availability of S-adenosylmethionine (SAM) leading to DNA hypomethylation [24-26]. Alternatively, the common oxygen radical adduct 8hydroxyguanine in DNA can dramatically inhibit the process of DNA methylation, so free radical damage may explain the decreased Alu methylation [27-30]. Possibly, high glucose concentration-induced cytosolic $\mathrm{Ca} 2+$ and ERK1/2 activation increases mitochondrial fragmentation and reactive oxygen species (ROS) levels [31-33]. Hence, hyperglycemia could promote cellular senescent formation by metabolic changes and tissue damage from oxidative stress resulting in increased free radicals that could lead to genomic hypomethylation and genomic instability in type 2 DM [34].

\section{Conclusions}

Our results not only showed significant Alu hypomethylation levels in type $2 \mathrm{DM}$ but also its association with high blood pressure. Therefore, Alu methylation is a promising biomarker for monitoring type $2 \mathrm{DM}$ complications. Moreover, Alu elements should be considered as epigenome-editing targets for future research in DM disability prevention and treatment.

\section{Additional file}

Additional file 1: Figure S1. Alu methylation patterns of COBRA-Alu assay. (A) The Alu amplicons are 133 bp and contain 2 CpG-dinucleotides. (B) A schematic representation of the COBRA-Alu assay shows the methylation patterns of Alu amplicons, including fully methylated loci ( $\mathrm{mCmC}$ ), unmethylated loci (uCuC) and two partially methylated forms (mCuC and $\mathrm{u}(\mathrm{mC})$. (C) For bisulfate treatment, methylated cytosine bases are not changed to uracil bases, whereas the unmethylated cytosine bases are converted to uracil bases. After the PCR products are digested with Taq1 restriction enzyme, the digested products are $\mathrm{mCmC}(43,32$ and $58 \mathrm{bp}), \mathrm{uCuC}(133 \mathrm{bp}), \mathrm{mCuC}$ ( 43 and $90 \mathrm{bp}$ ) and $\mathrm{uCmC}$ ( 75 and $58 \mathrm{bp}$ ). (TIFF $98 \mathrm{~kb}$ )

\section{Abbreviations}

COBRA: Combined Bisulfite Restriction Analysis; DM: Diabetes mellitus; FBS: Fasting blood sugar; HbA1C: Hemoglobin A1c; ROS: Reactive oxygen species; SAM: S-adenosylmethionine; WBCs: White blood cells

\section{Acknowledgements}

We thank the staff of the Ban Ton Riang Tambon Health Promoting Hospital, Nakhon Si Thammarat, Thailand, for their assistance in sample collection. 


\section{Funding}

This study was financially supported by the Institute of Research and Development Walailak University Grant No. WU58310 and Thailand Research Fund (TRF) Grant No. MRG5980090. This work was supported by the Thailand Research Fund Grant No. DPG5980005.

\section{Availability of data and materials}

Not application.

\section{Authors' contributions}

JT isolated DNA from plasma samples and processed the data. JT and MP performed the bisulfite DNA modification. JT and AM wrote the first draft of the manuscript. All authors read and approved the final manuscript.

\section{Ethics approval and consent to participate}

The study was reviewed and approved by the Ethics Clearance Committee on Human Rights Related to Researched Involving Human Subjects, Walailak University, Nakhon Si Thammarat, Thailand. Written informed consent was obtained from each participant.

\section{Consent for publication}

Not applicable.

\section{Competing interests}

The authors declare that they have no competing interests.

\section{Publisher's Note}

Springer Nature remains neutral with regard to jurisdictional claims in published maps and institutional affiliations.

\section{Author details}

'School of Medicine, Walailak University, Nakhon Si Thammarat, Thailand. ${ }^{2}$ Inter-Department Program of Biomedical Sciences, Faculty of Graduate School, Chulalongkorn University, Bangkok, Thailand. ${ }^{3}$ Center for Excellence in Molecular Genetics of Cancer and Human Diseases, Chulalongkorn University, Bangkok, Thailand. ${ }^{4}$ Department of Anatomy, Faculty of Medicine, Chulalongkorn University, Bangkok, Thailand.

\section{Received: 28 April 2017 Accepted: 24 August 2017}

\section{Published online: 31 August 2017}

\section{References}

1. Alberti K, Eckel RH, Grundy SM, Zimmet PZ, Cleeman II, Donato KA, Fruchart J-C, James WPT, Loria CM, Smith SC. Harmonizing the metabolic syndrome: a joint interim statement of the International Diabetes Federation Task Force on Epidemiology and Prevention; National Heart, Lung, and Blood Institute; American Heart Association; World Heart Federation; International Atherosclerosis Society; and International Association for the Study of Obesity. Circulation. 2009;120(16):1640-5.

2. Wang G. Raison d'être of insulin resistance: the adjustable threshold hypothesis. J R Soc Interface. 2014;11(101):20140892.

3. Peer N, Kengne A-P, Motala AA, Mbanya JC. Diabetes in the Africa Region: an update. Diabetes Res Clin Pract. 2014;103(2):197-205.

4. Lyssenko V, Laakso M. Genetic screening for the risk of type 2 diabetes: worthless or valuable? Diabetes Care. 2013;36(Supplement 2):S120-6.

5. Giacco F, Brownlee M. Oxidative stress and diabetic complications. Circ Res. 2010;107(9):1058-70.

6. Leong A, Porneala B, Dupuis J, Florez JC, Meigs JB. Type 2 diabetes genetic predisposition, obesity, and all-cause mortality risk in the US: a multiethnic analysis. Diabetes Care. 2016;39(4):539-46.

7. Fuchsberger C, Flannick J, Teslovich TM, Mahajan A, Agarwala V, Gaulton KJ, Ma C, Fontanillas P, Moutsianas L, McCarthy DJ. The genetic architecture of type 2 diabetes. Nature. 2016;536(7614):41-47.

8. Palmer AK, Tchkonia T, LeBrasseur NK, Chini EN, Xu M, Kirkland JL. Cellular senescence in type 2 diabetes: a therapeutic opportunity. Diabetes. 2015; 64(7):2289-98.

9. Testa R, Ceriello A. Pathogenetic loop between diabetes and cell senescence. Diabetes Care. 2007:30(11):2974-5.

10. Jintaridth $P$, Mutirangura A. Distinctive patterns of age-dependent hypomethylation in interspersed repetitive sequences. Physiol Genomics. 2010;41(2):194-200.
11. Jintaridth $P$, Tungtrongchitr $R$, Preutthipan $S$, Mutirangura $A$. Hypomethylation of Alu elements in post-menopausal women with osteoporosis. PloS one. 2013;8(8):1-8.

12. Batzer MA, Deininger PL. Alu repeats and human genomic diversity. Nat Rev Genet. 2002;3(5):370-9.

13. Deininger $\mathrm{PL}$, Moran JV, Batzer MA, Kazazian HH. Mobile elements and mammalian genome evolution. Curr Opin Genet Dev. 2003;13(6):651-8.

14. Terry MB, Delgado-Cruzata L, Vin-Raviv N, Wu HC, Santella RM. DNA methylation in white blood cells: association with risk factors in epidemiologic studies. Epigenetics. 2011;6(7):828-37.

15. Rerkasem K, Rattanatanyong P, Rerkasem A, Wongthanee A, Rungruengthanakit K, Mangklabruks A, Mutirangura A. Higher Alu methylation levels in catch-up growth in twenty-year-old offsprings. PLoS One. 2015;10(3):e0120032.

16. Jung M, Pfeifer GP. Aging and DNA methylation. BMC Biol. 2015:13(1):7.

17. López-Otín C, Blasco MA, Partridge L, Serrano M, Kroemer G. The hallmarks of aging. Cell. 2013:153(6):1194-217.

18. Pobsook T, Subbalekha K, Sannikorn P, Mutirangura A. Improved measurement of LINE-1 sequence methylation for cancer detection. Clin Chim Acta. 2011:412(3):314-21.

19. Wangsri S, Subbalekha K, Kitkumthorn N, Mutirangura A. Patterns and possible roles of LINE-1 methylation changes in smoke-exposed epithelia. PLoS One. 2012;7(9):e45292.

20. Nakkuntod J, Sukkapan P, Avihingsanon Y, Mutirangura A, Hirankarn N. DNA methylation of human endogenous retrovirus in systemic lupus erythematosus. J Hum Genet. 2013:58(5):241-9.

21. Harvey A, Montezano AC, Touyz RM. Vascular biology of ageing -implications in hypertension. J Mol Cell Cardiol. 2015;83:112-21.

22. Minamino T, Komuro I. Vascular cell senescence. Circ Res. 2007;100(1):15-26.

23. Benayoun BA, Pollina EA, Brunet A. Epigenetic regulation of ageing: linking environmental inputs to genomic stability. Nat Rev Mol Cell Biol. 2015; 16(10):593-610.

24. Kloypan C, Srisa-art M, Mutirangura A, Boonla C. LINE-1 hypomethylation induced by reactive oxygen species is mediated via depletion of $\mathrm{S}$ adenosylmethionine. Cell Biochem Funct. 2015;33(6):375-84

25. Anier K, Zharkovsky A, Kalda A. S-adenosylmethionine modifies cocaineinduced DNA methylation and increases locomotor sensitization in mice. Int J Neuropsychopharmacol. 2013;16(9):2053-66

26. Gören JL, Stoll AL, Damico KE, Sarmiento IA, Cohen BM. Bioavailability and lack of toxicity of S-adenosyl-L-methionine (SAMe) in humans. Pharmacotherapy. 2004;24(11):1501-7.

27. Shi Y, Hu FB. The global implications of diabetes and cancer. Lancet. 2014 383(9933):1947-8

28. Weitzman SA, Turk PW, Milkowski DH, Kozlowski K. Free radical adducts induce alterations in DNA cytosine methylation. Proc Natl Acad Sci. 1994; 91(4):1261-4.

29. Turk PW, Laayoun A, Smith SS, Weitzman SA. DNA adduct 8-hydroxyl-2'deoxyguanosine (8-hydroxyguanine) affects function of human DNA methyltransferase. Carcinogenesis. 1995;16(5):1253-5.

30. Tucker KL. Methylated cytosine and the brain: a new base for neuroscience. Neuron. 2001;30(3):649-52.

31. Yu T, Robotham $J L$, Yoon Y. Increased production of reactive oxygen species in hyperglycemic conditions requires dynamic change of mitochondrial morphology. Proc Natl Acad Sci U S A. 2006;103(8):2653-8.

32. Brem H, Tomic-Canic M. Cellular and molecular basis of wound healing in diabetes. J Clin Invest. 2007;117(5):1219-22.

33. Shah GN, Morofuji Y, Banks WA, Price TO. High glucose-induced mitochondrial respiration and reactive oxygen species in mouse cerebral pericytes is reversed by pharmacological inhibition of mitochondrial carbonic anhydrases: implications for cerebral microvascular disease in diabetes. Biochem Biophys Res Commun. 2013:440(2):354-8.

34. Attia S, Helal G, Alhaider A. Assessment of genomic instability in normal and diabetic rats treated with metformin. Chem Biol Interact. 2009;180(2):296304. 